\title{
Spatially Explicit Subpixel-Based Study On The Expansion of Artificial Impervious Surfaces And Its' Impacts On Soil Organic Carbon
}

\section{Yan Yan}

Luoyang Normal University

Weige Zhang ( $\square$ zhangwg@lynu.edu.cn )

Luoyang Normal University

\section{Yunfeng $\mathrm{Hu}$}

Institute of Geographic Sciences and Natural Resources Research

Huaipeng Liu

Luoyang Normal University

Xiaoping Zhang

Luoyang Normal University

\section{Yongxin Zhang}

Luoyang Normal University

\section{Research Article}

Keywords: artificial impervious surfaces, soil organic carbon, spatial analysis, urbanization, Kaifeng

Posted Date: November 22nd, 2021

DOI: https://doi.org/10.21203/rs.3.rs-867130/v1

License: (c) (1) This work is licensed under a Creative Commons Attribution 4.0 International License. Read Full License 
1 Spatially Explicit Subpixel-Based Study on the Expansion of Artificial Impervious Surfaces and Its'

\title{
Impacts on Soil Organic Carbon
}

Yan Yan ${ }^{\text {a }}$, Weige Zhang ${ }^{a^{*}}$, Yunfeng Hu ${ }^{\mathrm{b}}$, Huaipeng Liu ${ }^{\mathrm{a}}$, Xiaoping Zhang a and Yongxin Zhang ${ }^{\mathrm{c}}$

a School of Land and Tourism, Luoyang Normal University, Luoyang 471934, Henan Province, China

${ }^{b}$ Institute of Geographic Sciences and Natural Resources Research, Chinese Academy of Sciences, Beijing

100101, China

${ }^{c}$ School of Information Technology, Luoyang Normal University, Luoyang 471934, Henan Province, China

* Corresponding author. W. Zhang; E-mail address: zhangwg@lynu.edu.cn

\begin{abstract}
Precise spatiotemporal datasets of artificial impervious surfaces (AISs) are essential for evaluating urbanization processes and associated soil organic carbon (SOC) dynamics. However, spatially explicit studies on SOC stocks based on high-quality AIS data remain deficient, which affects the accuracy of urban SOC budgets. In this study, we used 30-m Landsat images and a subpixel-based model to accurately evaluate and quantify the annual AIS of Kaifeng, an ancient city in China that experienced intensive urbanization from 2000 to 2020. Soil organic carbon (SOC) dynamics were further estimated and spatially exhibited based on the SOC densities (SOCD) of different land covers observed in the field. Our results demonstrate that Kaifeng experienced drastic AIS expansion from 2000-2020, both in total area (an increase of $\sim 154.35 \%$ ) and density (described by mean AIS abundance, 0.56 vs. 0.72). Spatially, AIS mainly sprawled to the west, and infilling was observed in the old town. Moreover, the expansion of AIS in Kaifeng has resulted in a total of $0.08 \mathrm{Tg}$ of SOC loss over the past 20 years, and the study area has acted as a clear carbon source. The greatest SOC losses occurred during 2010 - 2015, mainly in the west — with >30\% ( $\sim 0.024 \mathrm{Tg}$ ) of the total loss occurring between 2010 and 2015 . This study provides new insights into urban growth through the mapping of growth patterns in terms of both outward sprawl and infill. We also provide a novel means of presenting the spatial patterns of urbanization-induced SOC dynamics using subpixel AIS maps.
\end{abstract}

Keywords: artificial impervious surfaces; soil organic carbon; spatial analysis; urbanization; Kaifeng; 


\section{Introduction}

Urbanization has become the main theme of global land change and is the foremost factor affecting the carbon cycle at multiple scales (Hutyra et al., 2014; Zhu et al., 2019). The most direct evidence of urbanization is the conversion of agriculture and/or natural lands into artificial environments and the sealing soils with artificial impervious surfaces (AISs). Generally, AIS is composed of materials that prevent the natural infiltration of water into soils and include building roofs, cement squares, and road surfaces (Zhu et al., 2019). The total global area of AIS reached 797,076 $\mathrm{km}^{2}$ in $2018-1.5$ times that in 1990 (Gong et al., 2020). The installation of AIS includes the removal of vegetation and organic-rich topsoil and the sealing of soils with impermeable materials, all of which can substantially, and both directly and indirectly, influence soil organic carbon (SOC) stocks (Lu et al., 2020; Piotrowska-Dlugosz and Charzynski, 2015; Zhao et al., 2012).

Although it is well known that SOC dynamics are closely related to spatial and temporal changes in AIS, spatially explicit studies on how AIS expansion affects SOC stocks remain rare and insufficient (Yan et al., 2015; Yan et al., 2016). Furthermore, estimates of the quantities and distributions of urban SOC budgets are

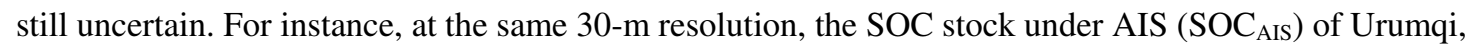
China in 2010 was $3.56 \mathrm{Tg}$, based on the work of Gong et al. (2020), who used pixel-based data; meanwhile, it was only $0.94 \mathrm{Tg}$ according to Zhang et al. (2015), who used subpixel-based data from the same year. The reason for the pronounced disparity between these results is that urban land cover is highly heterogeneous, such that 30-m pixels may contain more than two different land-cover types, such as AIS and vegetated areas (Lu and Weng, 2004, 2006; Zhang et al., 2015), thus constituting mixed pixels. Different solutions to the mixed pixel issue can result in large differences among the assessment results. Considering the large proportion of AIS in urban areas $(>50 \%)$ and the finer-scale resolution of subpixel datasets, AIS approaches that employ such data may be more accurate and appropriate for city-scale studies (Li et al., 2020; Wang and Li, 2019).

The temporal resolution of several public AIS datasets (5-10-year intervals) was found to be insufficient when attempting to reveal gradual changes in AIS and associated SOC dynamics (Schott et al., 2016; Zhu et al., 2020). Urban lands are highly dynamic and can undergo subtle changes over a relatively short period, and gradual changes in AIS expansion have been difficult to capture over longer observation periods (5-10 years apart) (Fu et al., 2019; Li et al., 2018). Meanwhile, small changes in AIS (i.e., excavation of foundations for tall buildings) could significantly disturb SOC stocks (Hu et al., 2018), making precise AIS datasets with dense frequencies essential for achieving a detailed understanding of changes in urbanization and for 
clarifying the effects of urbanization on local SOC pools. The limited availability of information on $\mathrm{SOC}_{\mathrm{AIS}}$ is a persistent knowledge gap in our understanding of the ecological effects of urbanization (Hutyra et al., 2014; Vasenev et al., 2018). Organic carbon sequestration is a vital ecosystem service performed by urban soils (Setälä et al., 2016). However, various land use activities in urban areas have resulted in significant differences among different land covers (Pouyat et al., 2002), for which $\mathrm{SOC}_{\mathrm{AIS}}$ is not yet fully understood (Dorendorf et al., 2015; Pouyat et al., 2006).

The expansion of AIS results in the occupation of former croplands and forests and the sealing of large areas of soil (Bren d'Amour et al., 2017; Scalenghe and Marsan, 2009). Since the SOC hidden beneath urban AIS greatly impacts the $\mathrm{C}$ budgets of urban ecosystems, many researchers have attempted to quantify their characteristics to develop an initial understanding of SOC $_{\mathrm{AIS}}$ (Table S1) (Vasenev and Kuzyakov, 2018). Nevertheless, the SOC density under AIS (SOCD $\left.{ }_{\text {AIS }}\right)$ varies remarkably among different cities. For example, the $\mathrm{SOCD}_{\mathrm{AIS}}$ at a depth of 0-100 $\mathrm{cm}$ in Lahti, Finland was only $1.2 \mathrm{~kg} \mathrm{C} \mathrm{m}^{-2}$ (Lu et al., 2020), while it was 9.6 $\mathrm{kg} \mathrm{C} \mathrm{m}{ }^{-2}$ in New York City, USA (Cambou et al., 2018), though a comparative analysis indicated that there was no significant difference between New York City and Paris, France at depths of 0-30 cm (Table S1) (Cambou et al., 2018). Whether or not there are significant differences among the SOCD $_{\text {AIS }}$ values of different cities has not been fully verified. Therefore, it is not appropriate to assume that SOCD $_{\text {AIS }}$ is equal to a fixed value when evaluating urbanization-induced SOC dynamics (Churkina et al., 2010; Pouyat et al., 2006). Additionally, urban SOC characteristics are also influenced by other factors, such as original land use/cover types, land-use history, urban functions, the intensity of development, and urban management (Puskás and Farsang, 2009; Vasenev and Kuzyakov, 2018). To fully understand the stock and dynamics of SOC in a specific city, $\mathrm{SOCD}_{\mathrm{AIS}}$ must be quantified based strictly on locally defined bulk densities and SOC contents.

In this study, we employed a subpixel approach to map the annual AIS of Kaifeng, China from 2000 to 2020 based on Landsat Thematic Mapper (TM), Enhanced Thematic Mapper Plus (ETM+), and Operational Land Imager (OLI) (TM/ETM+/OLI) images to demonstrate the gradual changes in AIS expansion in a spatially explicit manner, reveal its impacts on SOC, and locate the $\mathrm{C}$ sink/source during urbanization. The SOC dynamics were further calculated according to the field investigated SOCD data from different land covers, which were obtained from the literature. Our objectives were to (1) accurately quantify the temporal and spatial expansion of AIS at a finer scale; (2) reveal the spatial magnitude and dynamics of SOC in an urban area; and (3) develop a method for estimating and spatially presenting AIS expansion-induced SOC dynamics at the city scale; common to each of these aims was the goal of reducing uncertainty when estimating the impacts of AIS expansion on local/city SOC. 


\section{Materials and Methods}

\subsection{Study Area}

The city of Kaifeng $\left(34^{\circ} 11^{\prime}-35^{\circ} 01^{\prime} \mathrm{N}, 113^{\circ} 52^{\prime}-115^{\circ} 15^{\prime} \mathrm{E}\right)$ is located in the east-central province of Henan, China. With a built-up area of $151 \mathrm{~km}^{2}$ and a population of 4.57 million by the end of 2019, Kaifeng is one of the core development areas in the "Central Plains Urban Agglomeration Development Plan" issued by the National Development and Reform Commission (Wang and Liu, 2018) (Fig. 1). Kaifeng is also one of the most famous historical and cultural cities, known as the "ancient capital of the eight dynasties" with a history dating back more than 4,100 years (Storozum et al., 2020). Kaifeng is characterized by a typical warm temperate continental monsoon climate with four distinct seasons. The mean annual temperature is $14.4^{\circ} \mathrm{C}$ and the total precipitation amount of is $668.3 \mathrm{~mm}$. The landscapes of Kaifeng consist of forests, croplands, and wetlands; the main plant types are willows, locusts, and Paulownia, which can also be found in the metropolitan area. The main soil types are fluvio-aquatic and alluvium. Due to flooding of the Yellow River, several ancient capitals and cities are buried below the modern city of Kaifeng, at depths of 3-12 m; this creates a peculiar landscape of city accumulation and further influences soil development. Soils in Kaifeng have been exposed to high-intensity human activities for millennia because of the long history of habitation in this area; such history provides a typical and representative area in which to study the impacts on SOC stocks stemming from human activities. However, changes in AIS and their impacts on SOC stocks have rarely been examined.

\subsection{Methods}

This study involved three steps: (1) mapping annual AIS from Landsat TM/ETM+/OLI images and characterizing spatiotemporal changes in the AIS in Kaifeng from 2000-2020; (2) collecting and reanalyzing the SOCD of different land-cover types; and (3) estimating and displaying the impacts of AIS on SOC stocks in a spatially explicit manner (Fig. 2).

\subsubsection{Mapping AIS}

Annual Landsat TM, ETM+, and OLI data from 2000-2020 were collected to map the AIS of Kaifeng. A total of 20 Landsat images were used in this study, the details for which can be found in Table S2. All collected data had high geometric accuracy and were transformed into Universal Transverse Mercator (zone $\left.50^{\circ} \mathrm{N}\right)$. Radiometric calibration was applied to transform the digital number into reflectance values. Quick atmospheric corrections were then conducted to eliminate the influence of atmospheric absorption and 
scattering. To facilitate the selection of endmembers, the minimum noise fraction (MNF) method was used to determine the intrinsic noise of each image, and to ensure that the primary information was concentrated in the first three or four bands.

Endmember collection is a key step in the subpixel approach employed here. An endmember, which is distinguished from the mixed pixels, represents pixels that contain only the spectral information of one land cover type. Ideally, endmembers of a certain land cover are distributed at the top of the triangle generated by the different MNF bands. With the support of high-resolution datasets (collected from Google Earth v. 7.3.3, Google LLC, USA), the endmembers from four groups, which represented the four land-cover types of highalbedo objects, low-albedo objects, green vegetation, and bare soil, were collected from the two-dimensional scatter plots generated by the first three bands (Fig. S1).

A linear spectral mixture analysis (LSMA) model was employed to generate urban fractional land-cover maps. This is one of the main subpixel-based methods and is commonly used for extracting AIS from medium-resolution remote sensing data (Wang and Li, 2019). The LSMA model assumes that the spectrum of a single-pixel captured by a sensor is a linear combination of all components within that pixel (Equation 1,

Fig. S2).

$$
R_{i}=\sum_{k=1}^{n} f_{k} R_{i k}+\varepsilon_{k}
$$

where $i$ is the number of bands used, $k$ is the number of endmembers, such that $k=1,2, \ldots, n, R_{i}$ is the reflectance of band $i$, which may contain more than one endmember, $f_{k}$ is the abundance of endmember $k$ within a pixel, which represents the proportion of AIS within a single pixel and indicates the AIS density, $R_{i k}$ is the spectral reflectance of endmember $k$ in a single pixel on band $i$, and $\varepsilon_{k}$ is the error for band $i$. A fully constrained least-squares solution was then applied to unmix the remote sensing data into four fractional maps. The spatial AIS data were generated by taking the sum of fractional maps of the high-albedo and lowalbedo objects, according to the methods of Lu and Weng ( $\mathrm{Lu}$ and Weng, 2004). Finally, we eliminated nonimpervious regions based on the administrative boundaries of Kaifeng and global artificial impervious area (GAIA) data (Gong et al., 2020).

Due to the limitation that high-resolution images from 2000 were unavailable on Google Earth, we selected 2002 as the starting year. Reference images with 0.5-m resolutions were downloaded from Google Earth. As in a previous study (Gong et al., 2020), we randomly created 30 sampling plots with $4 \times 4$ pixels (i.e., $120 \mathrm{~m} \times 120 \mathrm{~m}$ ) for each of the representative years, which were taken to be 2002, 2005, 2010, 2015, and 2020, based on the aggregated AIS data (Fig. S3). For each year, these samples were geographically linked to the corresponding high-resolution images and the reference AIS was digitized. The percentage of 
AIS in each plot then was calculated (i.e., AIS/14400 m², e.g., Fig. S3f). We used the root mean square error

$$
R M S E=\sqrt{\frac{\sum_{i=1}^{n}\left(x_{r e f, i}-x_{\text {class }, i}\right)^{2}}{n}}
$$

$$
R=\frac{\sum_{i=1}^{n}\left(x_{\text {ref }, i}-\overline{x_{\text {ref. }}}\right)\left(x_{\text {class }, i}-\overline{x_{\text {class }}}\right)}{\sqrt{\sum_{i=1}^{n}\left(x_{\text {ref }, i}-\overline{x_{\text {ref. }}}\right)^{2}} \sqrt{\sum_{i=1}^{n}\left(x_{\text {class }, i}-\bar{x}_{\text {class }}\right)^{2}}},
$$

151

152

where $x_{r e f ., i}$ is the visually interpreted AIS abundance in plot $i, x_{r e f ., i}$ is the corresponding estimated AIS value in plot $I, \overline{x_{\text {ref. }}} \overline{x_{\text {class }}}$ is the average value of visually interpreted AIS abundance and the corresponding estimated AIS, and $n$ is the number of sampling plots $(n=30)$.

In this study, we focused only on urban expansion, while excluding the renewal process. Thus, AIS expansion was assessed based on the assumption that urban growth was irreversible. For the same pixel, if the value in the later periods was lower than the former, then the value of the former was given to the latter. The net gain in AIS was then used to reveal the expansion of AIS, which can be defined by Equation 4 as follows:

$$
\triangle A I S=A I S_{b}-A I S_{a}
$$

where $A I S_{a}$ and $A I S_{b}$ are the AIS areas at the beginning and end of the study period, respectively. The intensity of AIS expansion was then quantified as follows (Equation 5):

$$
K_{i}=\left(\sqrt[n]{A I S_{b} / A I S_{a}}-1\right) \times 100 \%
$$

where $K_{i}$ represents the annual growth rate of the AIS area, $A I S_{a}$ and $A I S_{b}$ are the same as in Equation (4), and $n$ represents the time period. The rate of change in AIS was estimated by the slope, $K$ (Equation 6 ). Where AIS represents the total AIS area in a certain year and $i$ is the number of years. Finally, we randomly extracted the values of 5000 points from the annual AIS map to analyze and thereby evaluate the changes in the urban form of Kaifeng from 2000 to 2020.

$$
K=\frac{n \times \sum_{i=1}^{n}\left(A I S_{i} \times i\right)-\sum_{i=1}^{n} A I S_{i} \sum_{i=1}^{n} i}{n \times \sum_{i=1}^{n} i^{2}-\left(\sum_{i=1}^{n} i\right)^{2}}
$$

\subsubsection{Assessing $S O C_{A I S}$ dynamics and locating carbon sources/sinks}


167

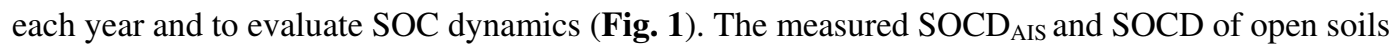

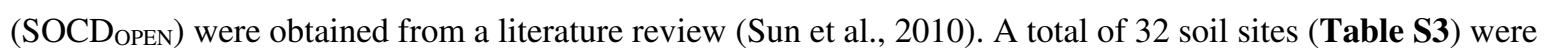
collected in 2009. Under the hypothesis that soil sealed by AIS is stable, the spatiotemporal pattern of SOC $_{\text {AIS }}$ from 2000 to 2020 (at 5-year intervals) was visualized and quantified based on spatially explicit AIS data. It must be noted that no change trajectory could be generated from the fractional AIS images; therefore, this study is mainly focused on SOC dynamics due to the expanded AIS occupying other land covers. Here, the

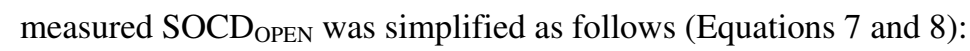

$$
S O C D_{O P E N}=\frac{n_{1} S O C D_{1}+n_{2} S O C D_{2}+\cdots+n_{i} S O C D_{k}}{n_{1}+n_{2}+\cdots+n_{i}}
$$

where $\bar{x}$ represents the weighted mean $\mathrm{SOCD}_{\mathrm{OPEN}}, x_{k}$ represents the SOCD of land-cover type $k, f$ is the number of sites taken by this land cover, $n$ is the sum of the sample points taken in the open soils, and SOCD $_{\text {AIS }}$ is the mean value estimated based on the soil sampling points located along roads and at buildings

(Table S3):

$$
\operatorname{SOCD}_{A I S}=\sum_{i=1}^{n}\left(x_{1}+x_{2}+\cdots+x_{n}\right) /_{n}
$$

where $\bar{x}$ represents the mean $\mathrm{SOCD}_{\mathrm{AIS}}$ and $x_{n}$ denotes the observed $\mathrm{SOCD}_{\mathrm{AIS}}$.

The SOC dynamics induced by AIS expansion were calculated similarly to the theory underlying LMSA, wherein the total SOC stock of a certain pixel was composed of $\mathrm{SOCD}_{\mathrm{AIS}}$ and $\mathrm{SOCD}_{\mathrm{OPEN}}$ (i.e., urban green space, bare lands, and croplands). The total SOC of this pixel could then be expressed as follows:

$$
S O C D=S O C D_{A I S} \times A I S+S O C D_{O P E N}
$$




$$
\triangle S O C=\left(\operatorname{SOCD}_{A I S} \times A_{A I S a}+\operatorname{SOCD}_{\text {OPEN }} \times A_{\text {OPENa }}\right)-\left(\operatorname{SOCD}_{A I S} \times A_{A I S b}+\operatorname{SOCD}_{\text {OPEN }} \times A_{O P E N b}\right)
$$

183

184

where $\triangle S O C$ is the change in SOC dynamics between two years, $A I S_{a}$ and $A I S_{b}$ are the same as in Equation

4, and $A_{O P E N a}$ and $A_{O P E N b}$ are the areas of the open soils at the beginning and end of the study period.

Because our research was based on the assumption that $\mathrm{SOCD}_{\mathrm{AIS}}$ and $\mathrm{SOCD}_{\mathrm{OPEN}}$ remain constant, Equation

10 could be simplified as follows:

$$
\triangle S O C=\left(S O C D_{A I S} \times\left(A_{A I S a}-A_{A I S b}\right)-S O C D_{O P E N} \times\left(A_{O P E N a}-A_{O P E N b}\right)\right.
$$

In this study, the reduced area of open soils was equal to the increase in AIS. Therefore, the SOC

dynamics could be simplified once more as:

$$
\triangle S O C D=\triangle A \times\left(S O C D_{A I S}-S O C D_{O P E N}\right)
$$

where $\Delta A=\left|A_{A I S a}-A_{A I S b}\right|$ or $\Delta A=\left|A_{\text {OPENa }}-A_{\text {OPENb }}\right|$ in Equation 12. The SOC dynamics were then spatially illustrated based on pixel-based results utilizing spatial analysis methods.

\section{Results}

\subsection{Assessment of AIS extraction accuracy}

Fig. 3 shows the relationship between the extracted AIS values from Landsat images and high-resolution data. All correlation coefficients $(R)$ were greater than $0.836(p<0.01)$ each year. Another indicator, RMSE, between the two datasets was further calculated, and the results showed that $R M S E<7.6 \%$. The major estimated error was less than $\pm 10 \%$, indicating that the results met the requirements of the follow-up study.

\subsection{AIS Dynamics from 2000-2020}

Kaifeng experienced a drastic expansion of AIS from 2000 to 2020, and newly developed AIS with a high growth rate $(>2.7 \%, p \leq 0.05)$ was mainly found in the western part of the metropolitan area (Fig. 4a). With an annual growth rate of $4.23 \%$, the total AIS area increased linearly by $\sim 154.35 \%(p<0.01)$ from 51.7 $\mathrm{km}^{2}$ in 2000 to $131.5 \mathrm{~km}^{2}$ in 2020 (Fig. 4b). The mean AIS abundance in Kaifeng clearly increased throughout the study period, having increased from 0.56 in 2000 to 0.72 in 2020 (Fig. 4). However, a different tendency was observed for the total area. Specifically, the mean AIS abundance grew rapidly until 2010, 
increasing from 0.56 in 2000 to 0.72 in 2010. After 2010, the rate of increase slowed and the AIS abundance remained stable, though a linear fit to the mean values shows a declining trend since 2010 (Fig. 4b).

According to the abundance of AIS (Fig. S4), the years 2005, 2010, 2015, and 2020 can be taken as time nodes to summarize the spatiotemporal changes in Kaifeng. From 2000 to 2015, AIS expanded at an accelerated rate (Fig. 5a-d). Specifically, the net gain in the AIS area increased from $15.56 \mathrm{~km}^{2}$ in 2005 (equal to 26.9\% of AIS area in 2000) to $16.30 \mathrm{~km}^{2}$ in 2010 (equal to $22.2 \%$ of AIS area in 2005) (Fig. 5f, g). The most intensive expansion accrued between 2010 and 2015, during which the areas of AIS expanded by $\sim 27.62 \mathrm{~km}^{2}$ in 2015, which was more than twice the AIS in 2000 (Fig. 5h). The spatial patterns of AIS expansion could be drawn as infilled old towns (main body of metropolitan area in 2000 in the east of Kaifeng), with most sprawl occurring to the west from 2000-2015 (Fig. 5f-h). The intensity and extent of the increase in AIS decreased after 2015 (Fig. 5e, i). The newly developed AIS was $15.03 \mathrm{~km}^{2}$, which was less than the increase observed from 2000-2005 (Fig. 5f, i). Spatial expansion was predominantly characterized by an infilling growth pattern based on the extent of expansion during the preceding period, which occurred throughout the main urban area. Moreover, the newly developed AIS was scattered and spatially discontinuous in the northwest, north, and southwest of the built-up area (Fig. 5j).

\subsection{SOC Dynamics caused by the Extension of AIS}

A total of $0.51 \mathrm{Tg}\left(1 \mathrm{Tg}=10^{12} \mathrm{~g}\right)$ of SOC was stored beneath the AIS in 2000, which had increased to 1.17 Tg by 2020 and had more than doubled since 2000 (Fig. 6a-f). It should be noted that the period from 2010-2015 exhibited the largest growth in $\operatorname{SOC}_{\mathrm{AIS}}(\sim 0.25 \mathrm{Tg})$, accounting for $\sim 31 \%$ of that in 2010 . Gains in $\mathrm{SOC}_{\mathrm{AIS}}$ were spatially consistent with the overall expansion of AIS, and were mainly concentrated in the western part of the metropolitan area Fig. 6i). However, the data indicated that $\mathrm{SOCD}_{\mathrm{AIS}}$ was lower than $\mathrm{SOCD}_{\mathrm{OPEN}}$ (Table S2). Gains in the $\mathrm{SOC}_{\mathrm{AIS}}$ stock also indicated SOC was lost during AIS expansion. As shown in Fig. 6, continuous SOC loss occurred with the expansion of AIS in Kaifeng since 2000.

From 2000 to 2020, a total of $0.08 \mathrm{Tg}$ of $\mathrm{C}$ was missing because of AIS expansion. Before 2010, the loss of SOC continued to increase and peaked in 2015 and then abated. Specifically, $0.02 \mathrm{Tg}$ of SOC and $0.015 \mathrm{Tg}$ of SOC were lost during the periods of 2000-2005 and 2005-2010, respectively (Fig. 6f). These two periods were dominated by slight losses in SOC that occurred throughout the study area, and small patches of strong SOC sources in the northwest (Fig. 6a, b). From 2010-2015, severe SOC loss occurred over a large area (Fig. 6c, g). The total SOC loss during this period was $0.024 \mathrm{Tg}$ (Fig. 6f) - approximately $31 \%$ of the total loss during the entire study period — and was mainly concentrated in northern and western Kaifeng (Fig. 6c). 
After 2015, the SOC loss driven by AIS installation was only 0.008 Tg (Fig. 6Error! Reference source not found.f), which was the lowest loss among each period, and was mainly distributed in the northwest of the city (Fig. 6d).

\section{Discussion}

\subsection{Changing characteristics of AIS in Kaifeng}

As the main type of land cover, it is crucial to understand the magnitude and spatial distribution of changes in AIS. The spatiotemporal patterns of AIS are closely related to urbanization-induced environmental problems, particularly when evaluating soil-related ecological problems, such as SOC losses in our study. Kaifeng has experienced dramatic growth since 2000 and has primarily sprawled to the west (Fig. 4). There are many reasons for this spatial change. First, Kaifeng was the old provincial capital of Henan, and the main part of the built-up area consisted of a high density of low-height buildings. This is also reflected in (Fig. 5ae), where Kaifeng is shown to have had a higher AIS density (histograms in figures) in the early years. Secondly, as these regions were difficult to renew for historical reasons, new lands were developed for urban expansion and the city could only expand to the east and west because of geographic restrictions. The Yellow River flows through the northern part of the city, and there are large areas of wetland resources, which the Chinese government has strictly prohibited from being used for urban development. To the south, the Lanzhou-Lianyungang Railway and military airfields cut off the possibility of southward expansion. Third, westward sprawling was the inevitable result (Fig. 5), not only because the current capital of Henan, Zhengzhou, lies to the west of Kaifeng, but also because of the implementation of the "Zhengzhou \& Kaifeng Integration" in the 13th five-year plan in 2005 (Liu et al., 2011). With the completion of the core region of the Zhengdong New District, the “Zhengzhou \& Kaifeng Integration” plan was substantially progressed in 2010, which further guided the extensive westward expansion of Kaifeng from 2010-2015 (Fig. 4, Fig. 5c-d). Additionally, it is notable that the land use intensity (taken as the AIS abundance in this study) in Kaifeng has remained high since 2010 (with mean values $>0.69$ in Fig. 4b), and the frequency of AIS abundances $>0.9$ has increased since 2010 (Fig. 5c-e) due to the unique land-related construction policies in Henan.

\subsection{Quantifying and locating SOC losses in Kaifeng}

Our study demonstrates that intensive urbanization (i.e., drastic AIS expansion) resulted in the loss of $\sim 0.67 \mathrm{Tg}\left(1.61 \mathrm{~kg} \mathrm{~m}^{-2}\right.$ ) of SOC in Kaifeng from 2000 to 2020, acted as a carbon source, and mainly occurred in the west (Fig. 6). The SOC loss was slightly higher than that found in Urumqi $\left(1.23 \mathrm{~kg} \mathrm{~m}^{-2}\right)$, a typical dryland city in NW China (Yan et al., 2016). Kaifeng is located in a warm temperate continental monsoon 
climate, so the background SOCD values were higher than those in Urumqi (i.e., $10.24 \mathrm{~kg} \mathrm{~m}^{-2} \mathrm{vs.} 9.77 \mathrm{~kg} \mathrm{~m}^{-2}$ of urban green space and $13.59 \mathrm{~kg} \mathrm{~m}^{-2} \mathrm{vs.} 5.59 \mathrm{~kg} \mathrm{~m}^{-2}$ of bare land, respectively). Kaifeng may suffer more SOC loss than Urumqi when exposed to construction activities since soils in temperate ecosystems have higher SOCD than dryland ecosystems. More importantly, AIS expansion in Kaifeng mainly occupied green spaces, the SOCD of which was much higher than $\operatorname{SOCD}_{\text {AIS }}\left(10.24 \mathrm{~kg} \mathrm{~m}^{-2} \mathrm{vs.} 8.88 \mathrm{~kg} \mathrm{~m}^{-2}\right)$ (Table S3), while in Urumqi, most displacement involved bare soils, which have similar SOCD and SOCD $_{\text {AIS }}$ values $(5.36 \mathrm{~kg}$ $\mathrm{m}^{-2}$ vs. $5.59 \mathrm{~kg} \mathrm{~m}^{-2}$ ) (Yan et al., 2016). This indicates that SOC loss due to conversion into AIS in Kaifeng may be stronger than in Urumqi.

In a review of the literature, we found that the SOCD of croplands was equal to that of AIS (Sun et al., 2010) (Table S3). According to China's second soil census data, Kaifeng is mainly composed of fluvio-aquic soil, and the average $100 \mathrm{~cm}$ depth SOCD was $\sim 5.17 \mathrm{~m}^{-2}$. This type of soil develops based on the river alluvium, which is loose (i.e., minimally compacted). According to Chinese construction standards (especially for roads), to achieve a certain degree of support, it was necessary to backfill a large amount of soil in Kaifeng. The soils used for backfilling may have had higher SOCD, which could have further increased the SOCD ${ }_{\text {AIS }}$ after consolidation. This may partly explain why the $\mathrm{SOCD}_{\mathrm{AIS}}$ in Kaifeng was higher. Even though SOCD $_{\text {AIS }}$ was higher, soil organic matter decomposed after being sealed (Majidzadeh et al., 2018) while accumulating in croplands (Zhang et al., 2018). Excluding croplands, $\mathrm{SOCD}_{\mathrm{AIS}}$ was the lowest among the different land covers (Table S3). The truth of AIS installation is that sealing the soil with impermeable materials blocks the exchange of water and energy between soils and the atmosphere (Scalenghe and Marsan, 2009). Therefore, AIS expansion both in the form of infilling and sprawl resulted in remarkable losses of SOC in Kaifeng from 2000 to 2020 (Fig. 6).

\subsection{How does $S O C D_{\text {AIS }}$ change in response to soil sealing?}

Sealed soils are usually assumed to be stable when evaluating the urban carbon cycle (Churkina et al., 2010; Kuittinen et al., 2016; Zhu et al., 2012). However, it has been noted that SOC $_{\text {AIS }}$ loss occurs in the first 53 years after sealing and tends to stabilize thereafter (Majidzadeh et al., 2018). In another study, it was reported that the top $20 \mathrm{~cm}$ of $\mathrm{SOC}_{\mathrm{AIS}}$ in Yixing showed a decreasing trend, and its variability could be characterized by $y=0.44+0.53 e^{-0.25}$ (Wei et al., 2014). Additionally, the potential carbon sequestration capacity of non-impervious regions (i.e., urban greenspaces and croplands) has also been easily overlooked in similar studies (Edmondson et al., 2012; Lu et al., 2020; Yan et al., 2016). Previous studies conducted in Kaifeng have also shown that the 100-cm-deep soils of the greenfield increased by $~ 66 \%$ between 1994 and 2006 (6.17 $\mathrm{kg} \mathrm{m}^{-2}$ in 1996 vs. $9.96 \mathrm{~kg} \mathrm{C} \mathrm{m}^{-2}$ in 2006), with an average annual growth rate of 4.07\% (Ma et al., 
1999; Sun et al., 2008) (Table S4). The 0-20-cm SOC of typical croplands in Henan has shown an increasing trend at a rate of $0.033 \mathrm{~kg} \mathrm{~m}^{-2} /$ year $\left(1.65 \mathrm{~m}^{-2}\right.$ in 1981 vs. $2.65 \mathrm{~m}^{-2}$ in 2011) (Zhang et al., 2018). Therefore, the impact of AIS installation on local SOC is not confined to the removal of topsoil, yet the mechanisms by which SOC changes after sealing have not been clearly addressed. Furthermore, the removal of vegetation has resulted in direct losses to aboveground biomass, and further destroyed the potential carbon sequestration of the original vegetation/soil, leading to the "invisible loss" of SOC. As the SOC dynamics caused by AIS expansion are very complex, the aforementioned issues should be fully considered in future studies.

\subsection{Strengths and uncertainties}

In this study, the annual growth of AIS in Kaifeng was demonstrated at the subpixel level. Changes in urban form were further delineated based on the abundance of AIS within a pixel. Additionally, we evaluated and spatially presented the SOC dynamics based on precise AIS and field data. Here, we provide insights into the monitoring of AIS expansion in terms of both sprawl and infilling, and delineate the trends in changes in AIS based on analyses of the proportions of AIS within a pixel. The latest global annual AIS data from 19852018 could improve our understanding of the gradual changes in AIS within a given city (Gong et al., 2020). Nonetheless, compared to our study, this dataset can only be used to understand AIS sprawl, to the exclusion of infilling, as it involves pixel-based data (Fig. S5). Likewise, most existing global urban products are pixelbased and have coarse temporal resolutions (He et al., 2019; Liu et al., 2018; Zhou et al., 2018). Meanwhile, urban environments are more dynamic than natural ecosystems (i.e., forests and grasslands), and can undergo many qualitative and subtle changes within a short period and at fine scales (Li et al., 2018). Therefore, highfrequency AIS data that can capture transient and gradual changes in urban development, such as the subpixel data used here, provide a means of comprehensively understanding urbanization-induced ecological issues, as well as more reliable information for urban management.

Here, we provide a framework to spatially illustrate SOC dynamics based on precise AIS data. Previous studies have mainly been focused on revealing the differences in SOC and/or soil nitrogen between AIS and pervious surfaces based on field data (Lu et al., 2020; Raciti et al., 2012), rather than on understanding SOC dynamics and AIS expansion in a spatially explicit way. We found that strong carbon sources exist in western Kaifeng, where drastic AIS expansion occurred; this information could help policymakers to: (1) take action to avoid generating strong carbon sources during continued western urbanization, and (2) enhance parks and improve greenspace coverage to compensate for SOC losses. Since rapid urbanization in the 21 st century is responsible for many ecological issues, and to meet China's promise to reach peak $\mathrm{CO}_{2}$ emissions before 

2030 and achieve carbon neutrality before 2060 (Normile, 2020), detailed information on the magnitude of SOC dynamics and location of carbon sinks/sources is needed to effectively implement carbon management policies and practices.

Some remaining uncertainties in this work should be addressed in the future. The first stems from the estimated $\mathrm{SOCD}_{\mathrm{AIs}}$. Generally, many randomized soil sample plots are required to calculate a confident SOCD value. However, it is difficult to collect soil samples beneath AIS because of the constraints of urban management regimes, which is a common issue in most studies concerned with soil properties under AIS (Table S1). We recognize that it is unconvincing to use limited data to assess the dynamics induced by AIS expansion. However, the essence of AIS expansion is the conversion of soil with a higher SOCD into soil with a lower organic carbon density beneath the AIS, which results in a considerable amount of SOC loss (Wei et al., 2014; Yan et al., 2015). Although this may present a source of great uncertainty, our results are consistent with those of previous studies (Lu et al., 2020; Yan et al., 2016), which suggests that the findings are robust across various methodologies. Another uncertainty is based on the assumption that the $\mathrm{SOC}_{\mathrm{AIS}}$ is stable after being sealed. While we found three reports related to the dynamics of $\mathrm{SOCD}_{\mathrm{AIS}}$ in the literature (Dou et al., 2021; Majidzadeh et al., 2018; Wei et al., 2014), we could not confirm whether or not these findings were applicable in our study. In conclusion, SOC dynamics during the expansion of AIS are subtle and more observed SOCD is needed. Various factors (e.g., measured SOCD of different land cover types, soil disturbance suffered during construction, $\mathrm{SOC}_{\mathrm{AIS}}$ dynamics, and the end of the removed soils) need to be considered in the calculation of SOC dynamics due to urbanization. In the future, we will deepen our analyses to help resolve these issues and better understand how AIS impact SOC.

\section{Acknowledgments}

We thank Ph. D Batu Nacun, Ph.D. Shihua Zhu, Ph.D. Xiuliang Yuan for providing valuable advice and comments in writing this article. Thank Lizi Xie for the help on the AIS data accuracy assessment.

\section{Decelerations}

Funding: This work was supported by the Natural Science Foundation of Henan Province of China, (Grant number 212300410212). 
351 Conflict of interest: The authors have no financial or proprietary interests in any material discussed in this 352 article.

353 Availability of data and material: Not applicable

354 Code availability: Not applicable

Author Contributions: All authors contributed to the study conception and design. Material preparation, data collection and analysis were performed by Yan Yan, Weige Zhang, and Yunfeng Hu. The first draft of the manuscript was written by Yan Yan. The final manuscript was checked and revised by Huaipeng Liu,

Xiaoping Zhang and Yongxin Zhang. All authors commented on previous versions of the manuscript. All authors read and approved the final manuscript.

\section{References}

Bren d'Amour, C., Reitsma, F., Baiocchi, G., Barthel, S., Güneralp, B., Erb, K.-H., Haberl, H., Creutzig, F., Seto, K. C., 2017, Future urban land expansion and implications for global croplands, Proceedings of the National Academy of Sciences 114(34):8939.

Cambou, A., Shaw, R. K., Huot, H., Vidal-Beaudet, L., Hunault, G., Cannavo, P., Nold, F., Schwartz, C., 2018, Estimation of soil organic carbon stocks of two cities, New York City and Paris, Science of The Total Environment 644:452-464.

Churkina, G., Brown, D. G., Keoleian, G., 2010, Carbon stored in human settlements: the conterminous United States, Global Change Biology 16(1):135-143.

Dorendorf, J., Eschenbach, A., Schmidt, K., Jensen, K., 2015, Both tree and soil carbon need to be quantified for carbon assessments of cities, Urban Forestry \& Urban Greening 14(3):447-455.

Dou, X., Lu, M., Chen, L., 2021, Comparison on soil organic carbon and nitrogen dynamics between urban impervious surfaces and vegetation, Authorea. 
Edmondson, J. L., Davies, Z. G., McHugh, N., Gaston, K. J., Leake, J. R., 2012, Organic carbon hidden in urban ecosystems, Scientific Reports 2.

Fu, Y., Li, J., Weng, Q., Zheng, Q., Li, L., Dai, S., Guo, B., 2019, Characterizing the spatial pattern of annual urban growth by using time series Landsat imagery, Science of The Total Environment 666:274-284.

Gong, P., Li, X., Wang, J., Bai, Y., Chen, B., Hu, T., Liu, X., Xu, B., Yang, J., Zhang, W., Zhou, Y., 2020, Annual maps of global artificial impervious area (GAIA) between 1985 and 2018, Remote Sensing of Environment 236:111510.

He, C., Liu, Z., Gou, S., Zhang, Q., Zhang, J., Xu, L., 2019, Detecting global urban expansion over the last three decades using a fully convolutional network, Environmental Research Letters 14(3):034008.

Hu, Y., Dou, X., Li, J., Li, F., 2018, Impervious Surfaces Alter Soil Bacterial Communities in Urban Areas: A Case Study in Beijing, China, Frontiers in Microbiology 9(226).

Hutyra, L. R., Duren, R., Gurney, K. R., Grimm, N., Kort, E. A., Larson, E., Shrestha, G., 2014, Urbanization and the carbon cycle: Current capabilities and research outlook from the natural sciences perspective, Earth's Future 2(10):473-495.

Kuittinen, M., Moinel, C., Adalgeirsdottir, K., 2016, Carbon sequestration through urban ecosystem services: A case study from Finland, Science of The Total Environment 563-564:623-632.

Li, W., Zhou, W., Bai, Y., Pickett, S. T. A., Han, L., 2018, The smart growth of Chinese cities: Opportunities offered by vacant land, Land Degradation \& Development 29(10):3512-3520.

Li, X., Gong, P., Zhou, Y., Wang, J., Bai, Y., Chen, B., Hu, T., Xiao, Y., Xu, B., Yang, J., Liu, X., Cai, W., Huang, H., Wu, T., Wang, X., Lin, P., Li, X., Chen, J., He, C., Li, X., Yu, L., Clinton, N., Zhu, Z., 2020 , Mapping global urban boundaries from the global artificial impervious area (GAIA) data, Environmental Research Letters 15(9):09444. 
Liu, X., Ding, C., Lu, C., 2011, Empirical Study on the Impact of Zhengzhou-Kaifeng Integration on House Prise of Kaifeng City (In Chinese), Areal Research and Development 30(3):52-55.

Liu, X., Hu, G., Chen, Y., Li, X., Xu, X., Li, S., Pei, F., Wang, S., 2018, High-resolution multi-temporal mapping of global urban land using Landsat images based on the Google Earth Engine Platform, Remote Sensing of Environment 209:227-239.

Lu, C., Johan, K. D., M., S. H., 2020, Soil sealing causes substantial losses in C and N storage in urban soils under cool climate, Science of The Total Environment 725:138369.

Lu, D. S., Weng, Q. H., 2004, Spectral mixture analysis of the urban landscape in Indianapolis with landsat ETM plus imagery, Photogrammetric Engineering and Remote Sensing 70(9):1053-1062.

Lu, D. S., Weng, Q. H., 2006, Use of impervious surface in urban land-use classification, Remote Sensing of Environment 102(1-2):146-160.

Ma, J., Zhang, L., Li, Y., 1999, Preliminary study on soil properties and pollution in Kaifeng City, Chinese Journal of Soil Science 30(2):93-96. In Chinese.

Majidzadeh, H., Lockaby, B. G., Price, R., Governo, R., 2018, Soil carbon and nitrogen dynamics beneath impervious Surfaces, Soil Science Society of America Journal 82(3):663-670.

Normile, D., 2020, China's bold climate pledge earns praise_-but is it feasible?, Science 370(6512):17-18.

Piotrowska-Dlugosz, A., Charzynski, P., 2015, The impact of the soil sealing degree on microbial biomass, enzymatic activity, and physicochemical properties in the Ekranic Technosols of Torun (Poland), Journal of Soils and Sediments 15(1):47-59.

Pouyat, R., Groffman, P., Yesilonis, I., Hernandez, L., 2002, Soil carbon pools and fluxes in urban ecosystems, Environmental Pollution 116, Supplement 1(0):S107-S118. 
Pouyat, R. V., Yesilonis, I. D., Nowak, D. J., 2006, Carbon storage by urban soils in the United States, Journal of Environmental Quality 35(4):1566-1575.

Puskás, I., Farsang, A., 2009, Diagnostic indicators for characterizing urban soils of Szeged, Hungary, Geoderma 148(3):267-281.

Raciti, S. M., Hutyra, L. R., Finzi, A. C., 2012, Depleted soil carbon and nitrogen pools beneath impervious surfaces, Environmental Pollution 164:248-251.

Scalenghe, R., Marsan, F. A., 2009, The anthropogenic sealing of soils in urban areas, Landscape and Urban Planning 90(1):1-10.

Schott, J. R., Gerace, A., Woodcock, C. E., Wang, S., Zhu, Z., Wynne, R. H., Blinn, C. E., 2016, The impact of improved signal-to-noise ratios on algorithm performance: Case studies for Landsat class instruments, Remote Sensing of Environment 185:37-45.

Setälä, H. M., Francini, G., Allen, J. A., Hui, N., Jumpponen, A., Kotze, D. J., 2016, Vegetation Type and Age Drive Changes in Soil Properties, Nitrogen, and Carbon Sequestration in Urban Parks under Cold Climate, Frontiers in Ecology and Evolution 4(93).

Storozum, M., Lu, P., Wang, S., Chen, P., Yang, R., Ge, Q., Cao, J., Wan, J., Wang, H., Qin, Z., Liu, H., Park, E., 2020, Geoarchaeological evidence of the AD 1642 Yellow River flood that destroyed Kaifeng, a former capital of dynastic China, Scientific Reports $\mathbf{1 0 .}$

Sun, Y., Ma, J., Li, C., 2008, Variations of the content and density of urban soil organic carbon in Kaifeng city, Journal of Henan University (Natural Science) 38(5):491-496. In Chines.

Sun, Y. L., Ma, J. H., Li, C., 2010, Content and densities of soil organic carbon in urban soil in different function districts of Kaifeng, Journal of Geographical Sciences 20(1):148-156. 
Vasenev, V., Kuzyakov, Y., 2018, Urban soils as hotspots of anthropogenic carbon accumulation: Review of stocks, mechanisms and driving factors, Land Degradation \& Development.

Vasenev, V. I., Stoorvogel, J. J., Leemans, R., Valentini, R., Hajiaghayeva, R. A., 2018, Projection of urban expansion and related changes in soil carbon stocks in the Moscow Region, Journal of Cleaner Production 170:902-914.

Wang, J., Liu, S., 2018, SWOT Analysis of the Development Planning of Central Plains Urban Agglomeration under the Background of New Era.

Wang, Y., Li, M., 2019, Urban impervious surface detection from remote sensing images: a review of the methods and challenges, IEEE Geoscience \& Remote Sensing Magazine 7(3):64-93.

Wei, Z., Wu, S., Yan, X., Zhou, S., 2014, Density and stability of soil organic carbon beneath impervious surfaces in urban areas, in: PloS one, pp. e109380.

Yan, Y., Kuang, W., Zhang, C., Chen, C., 2015, Impacts of impervious surface expansion on soil organic carbon - a spatially explicit study, Scientific Reports $\mathbf{5}$.

Yan, Y., Zhang, C., Hu, Y., Kuang, W., 2016, Urban land-cover change and Its impact on the ecosystem carbon storage in a dryland city, Remote Sensing 8(1):6.

Zhang, C., Chen, Y., Lu, D., 2015, Detecting fractional land-cover change in arid and semiarid urban landscapes with multitemporal Landsat Thematic mapper imagery, Mapping Sciences \& Remote Sensing 52(6):700722.

Zhang, C., Li, W., Zhao, Z., Zhou, Y., Zhang, J., Wu, Q., 2018, Spatiotemporal Variability and Related Factors of Soil Organic Carbon in Henan Province, Vadose Zone Journal 17(1):180109. 
457

458

459

460

461

462

463

464

465

466

467

468

Zhao, D., Li, F., Wang, R. S., Yang, Q. R., Ni, H. S., 2012, Effect of soil sealing on the microbial biomass, N transformation and related enzyme activities at various depths of soils in urban area of Beijing, China, Journal of Soils and Sediments 12(6):1004-1006.

Zhou, Y., Li, X., Asrar, G. R., Smith, S. J., Imhoff, M., 2018, A global record of annual urban dynamics (19922013) from nighttime lights, Remote Sensing of Environment 219:206-220.

Zhu, C., Zhao, S. Q., Zhou, D. C., 2012, Organic Carbon Storage in Urban Built-up Areas of China in 19972006, Chinese Journal of Applied Ecology 23(5):1195-1202.

Zhu, Z., Zhang, J., Yang, Z., Aljaddani, A. H., Cohen, W. B., Qiu, S., Zhou, C., 2020, Continuous monitoring of land disturbance based on Landsat time series, Remote Sensing of Environment 238:111116.

Zhu, Z., Zhou, Y., Seto, K. C., Stokes, E. C., Deng, C., Pickett, S. T. A., Taubenböck, H., 2019, Understanding an Urbanizing Planet: Strategic Directions for Remote Sensing, Remote Sensing of Environment 228:164182. 


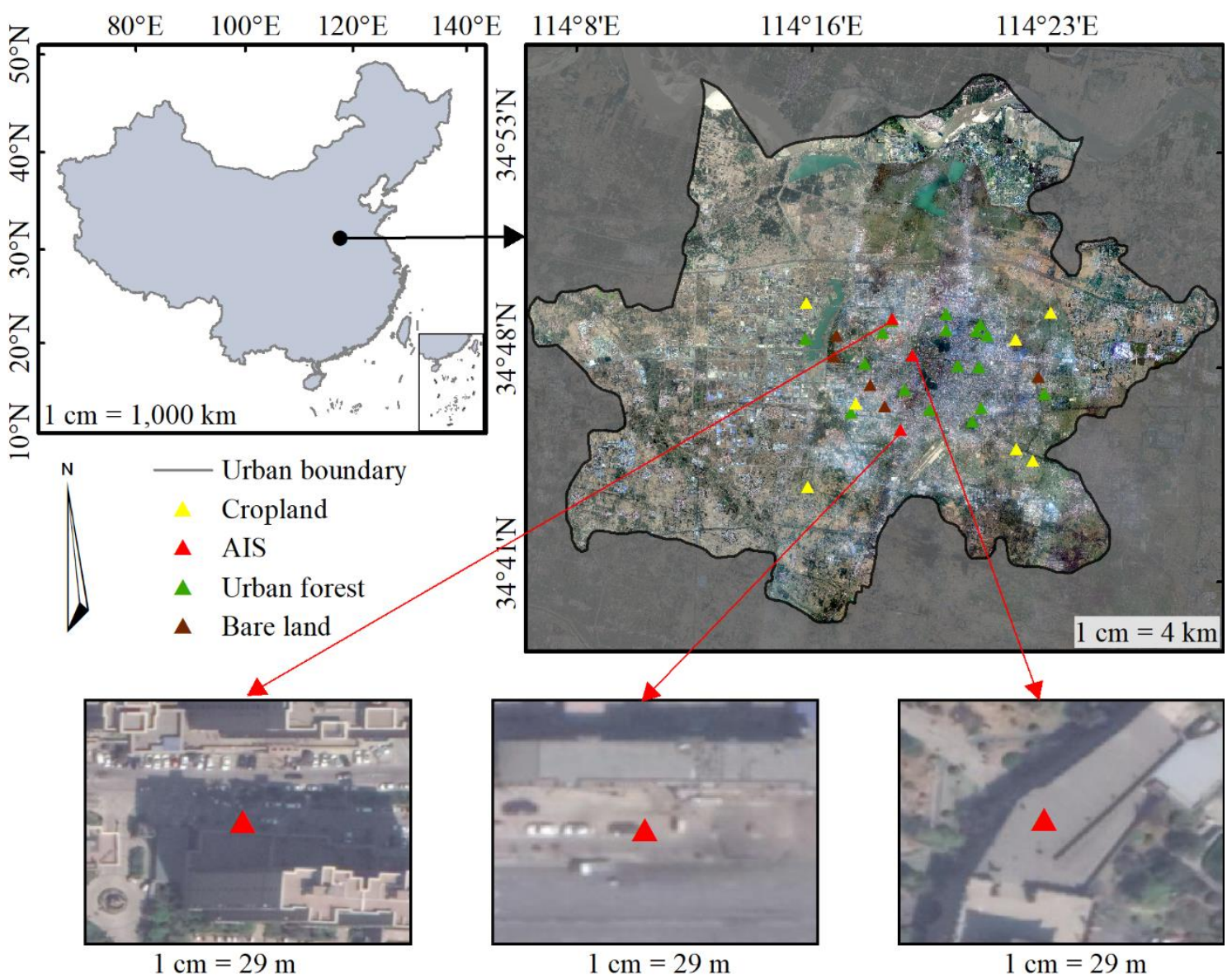

470 Fig. 1 Location of the study area and sampling sites. A WorldView-3 image (bands 5, 3, and 2) acquired on 471 May 26, 2020 at a 0.5-m spatial resolution was used 


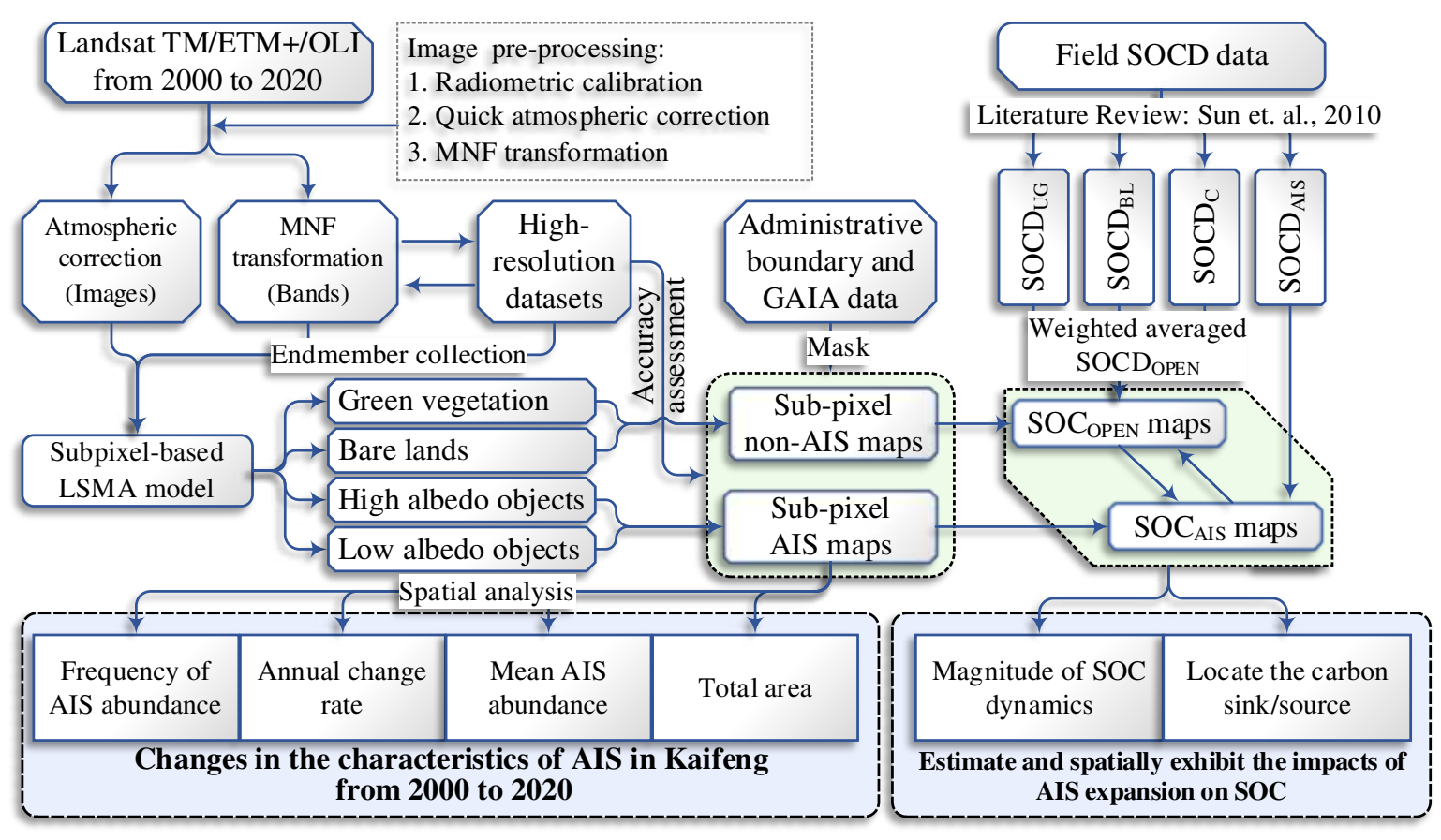

473 Fig. 2 Workflow of the study. Abbreviations: GAIA, global artificial impervious area; LMSA, linear spectral 474 mixture analysis; MNF, minimum noise fraction; $\mathrm{SOCD}_{\mathrm{BL}}$, $\mathrm{SOCD}$ of bare lands; $\mathrm{SOCD}_{\mathrm{C}}$, $\mathrm{SOCD}_{\mathrm{N}}$ of croplands; 475 SOCD ${ }_{\text {UG }}$, SOCD of urban green spaces 

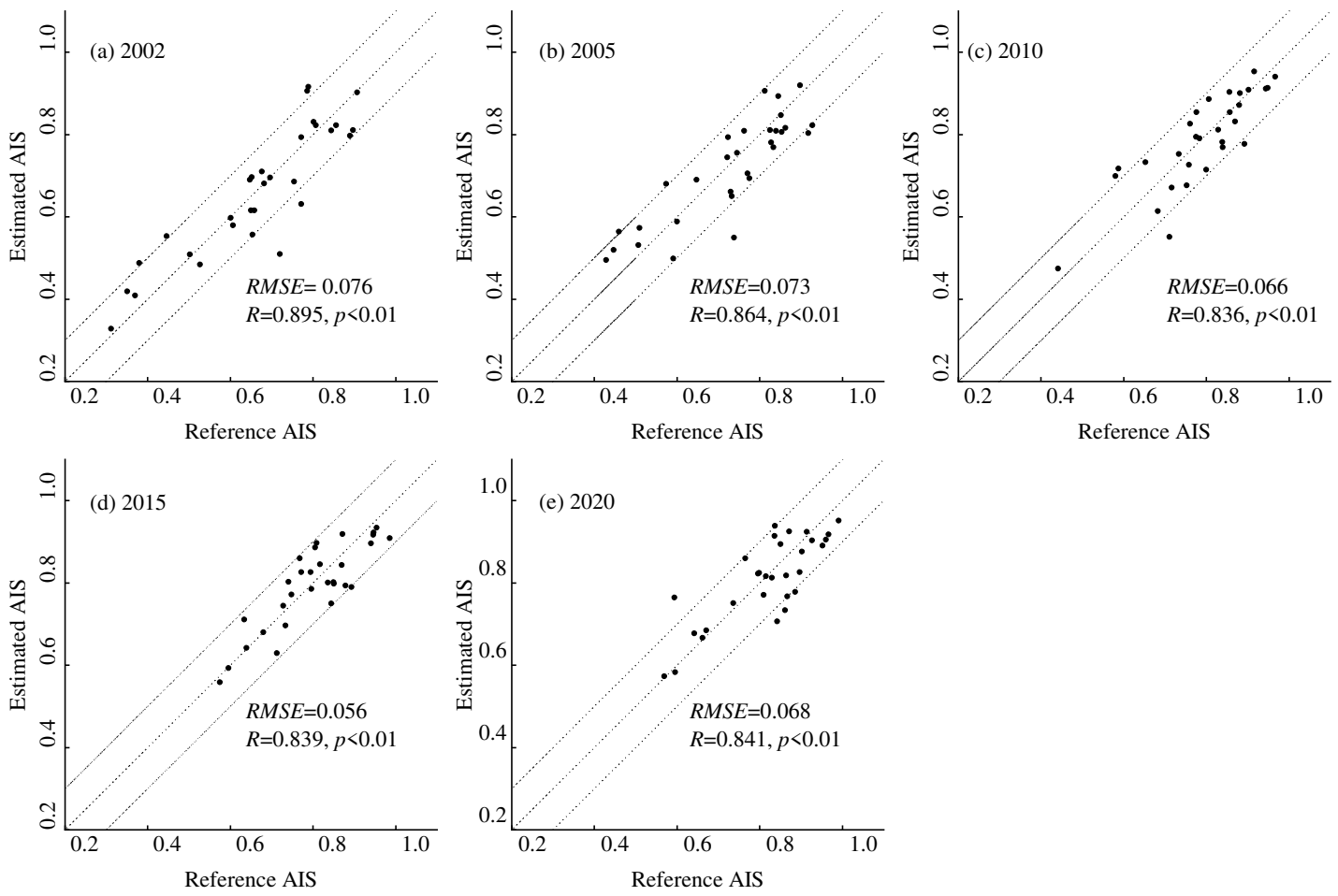

Fig. 3 Relationship between estimated fraction of AIS and reference data in Kaifeng from 2002-2020 


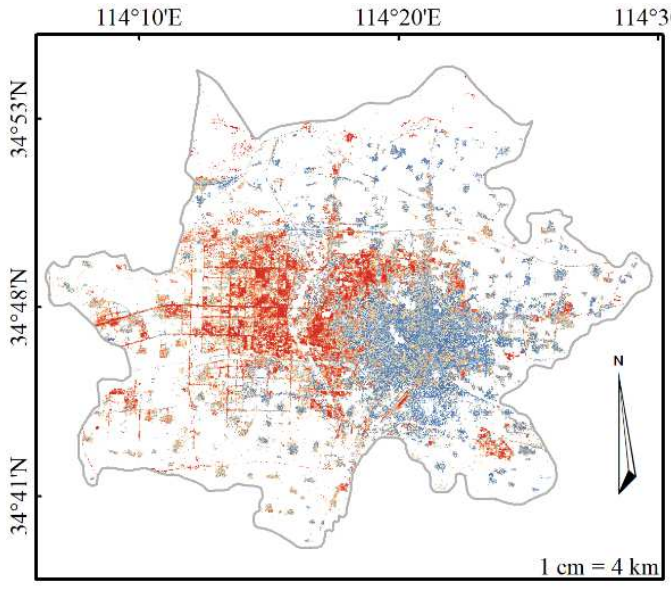

(a) Annual rate of change in AIS (\%) $\begin{array}{lllllllllll}0.00 & 0.38 & 0.58 & 0.74 & 0.90 & 1.10 & 1.13 & 1.16 & 1.19 & 2.70 & 7.50\end{array}$

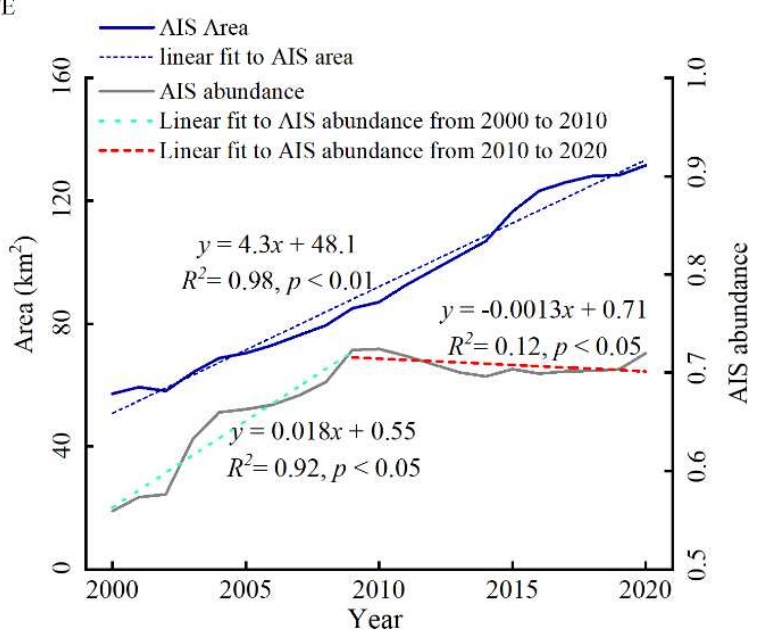

(b) Temporal patterns of total area and mean abundance of AIS

479 Fig. 4 Geographic distribution of changes in AIS (a) and temporal patterns of total AIS area and mean abundance (b) in Kaifeng from 2000-2020 


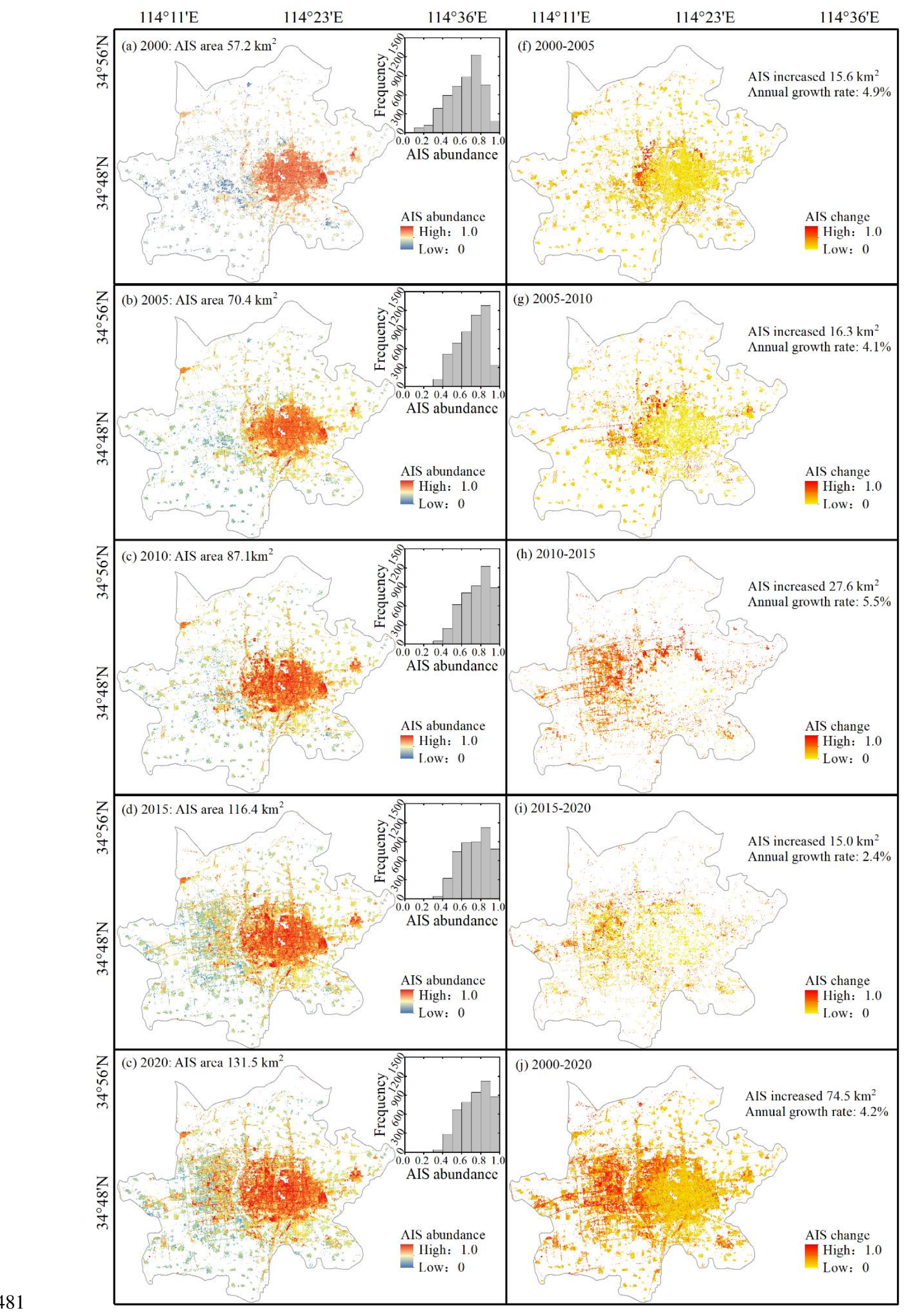




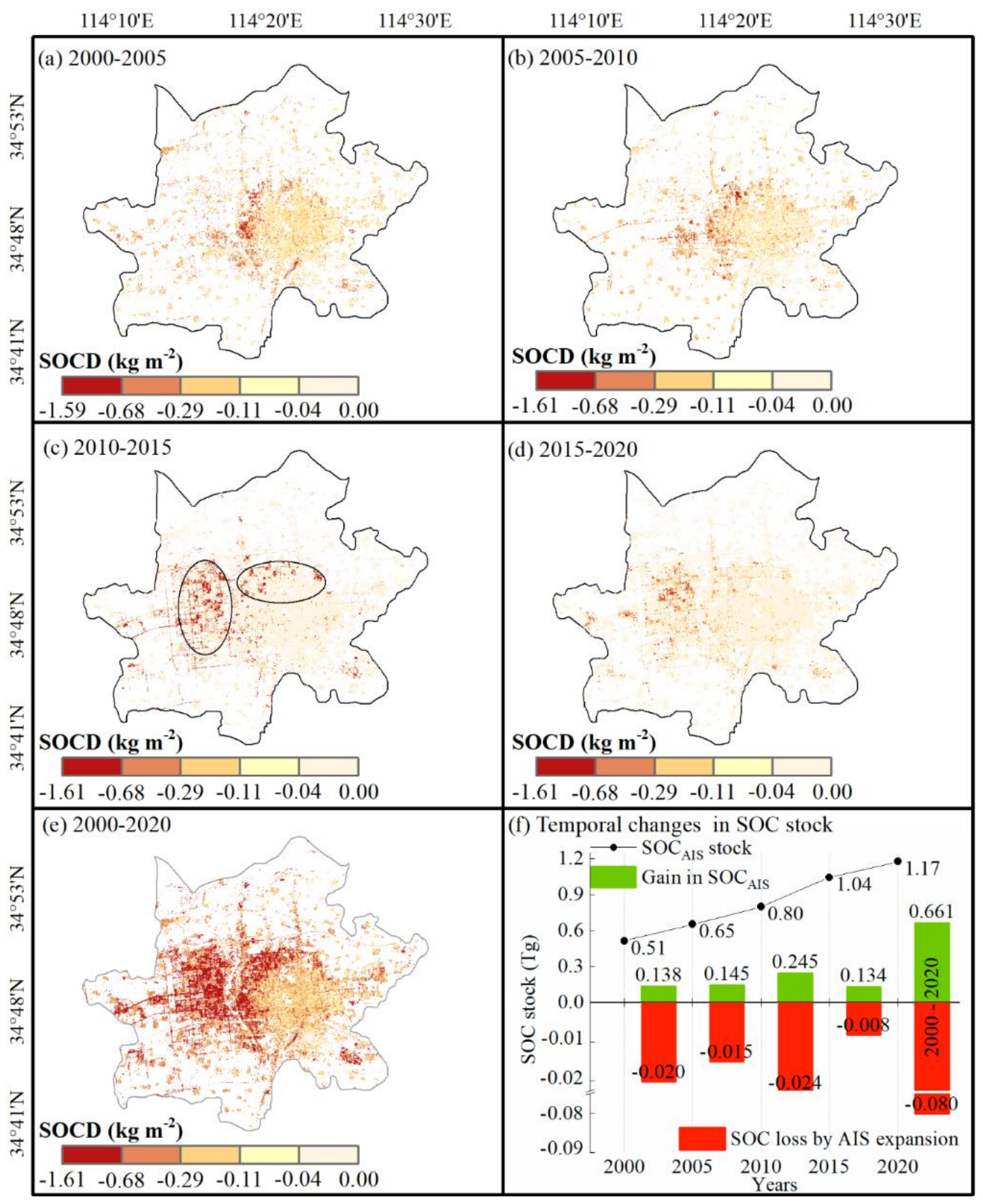

Fig. 6 Spatially explicit SOC dynamics influenced by the expansion of AIS in Kaifeng from 2000-2020 


\section{Supplementary Files}

This is a list of supplementary files associated with this preprint. Click to download.

- Supplementarymaterials.docx 